\title{
$1.38 \mu_{\mathbf{m}}$ 波长冰晶粒子的单次和 多次散射特性
}

\section{张国栋 许丽生 丁继烈}

(成都气象学院大气辐射与卫星遥感实验室. 成都 610041: 北京大学暴雨监测与预测国家重点实验室. 北京 100871)

摘要 研究了由六角形柱状和盘状冰晶组成的卷云在波长为 $1.38,3.979,6.5$ 和 $11.03 \mu_{\mathrm{m}}$ 的辐射特性与它的微物理特性的关系。卷云的单次散射用 Mie 散射理论和改进的射线光学理 论计算, 其辐射特性用累加-倍加法计算. 结果表明, 卷云在近红谱区的 $1.38 \mu_{\mathrm{m}}$, 相似性参数 及辐射特性与卷云的微物理特性之间的关系比当前气象业务卫星所使用的 3 个红外通道要 好. 因而, $1.38 \mu_{\mathrm{m}}$ 水汽吸收带在遥感卷云微物理特性及其光学特性方面具有潜在应用可能 性.

\section{关键词 冰晶粒子 遥感通道 单次散射 多次散射}

卷云的精确探测对云的气候学和大气辐射传输过程模式研究均具有重要的影响. 研究表 明, 目前的业务卫星, 例如, NOAA AVHRR, 很难对卷云进行精确的探测, 特别是在陆面 上. 因此, 一个重要的研究方向是寻找新的波长和发展新的遥感器. 最近, Gao 等人 ${ }^{[1]}$ 和 Hutchison 等人 ${ }^{[2]}$ 发现利用 $1.38 \mu_{\mathrm{m}}$ 水汽吸收带中心附近的窄通道探测卷云非常有效. 因此, 在未来的 MODIS( Moderate Resolution Imaging Spectrometer) 仪器中 ${ }^{[1,3]}$, 已建议增加一个 $1.375 \mu_{\mathrm{m}}$ 通道，以增强从空间探测卷云的能力，但文献 $[1,2]$ 只限于遥感通道的图象分析. 本文从卷云大气条件下的多重散射辐射传输理论出发, 计算和分析 $1.38 \mu_{\mathrm{m}}$ 波长冰晶粒子的 单次和多次散射特性, 揭示 $1.38 \mu_{\mathrm{m}}$ 水汽吸收带探测卷云的能力和本质.

为了研究卷云的卫星遥感, 需要知道卷云微物理学的两个基本参量, 即冰晶粒子的形状 和谱分布. 为此, 考虑随机取向的六角柱状和盘状冰晶, 和由两个修正的 $\Gamma$ 函数谱合成的谱 分布, 即

$$
\begin{aligned}
& n(r)=w n_{1}(r)+(1-w) n_{2}(r), \\
& n_{j}(r)=c_{j}^{-1} r_{j} \exp \left(-b_{j} r_{j^{j}}^{\mathrm{\gamma}}\right), \\
& b_{j}=\left(a_{j} / \gamma_{j}\right) r_{j}{ }^{\gamma}, \\
& \left.c_{j}=\overline{\gamma_{j}}{ }^{1} b_{j}^{-\left(a_{j}+1\right) / \gamma_{j}} \Gamma \Gamma\left(\alpha_{j}+1\right) / \gamma_{j}\right\rceil . i=1.2 .
\end{aligned}
$$

其中 $n_{1}$ 和 $n_{2}$ 分别表示小粒子和大粒子谱, $w$ 确定两种分布的相对丰度 $(0 \leqslant w \leqslant 1), \alpha_{j}$ 和 $\gamma_{j}$ 是谱形参数, $r_{j}$ 表示谱的峰值所对应的粒子大小. 为了比较真实地描述自然界中处于各种不 同发展阶段的卷云的微物理特征, 在公式(1)、(2) 的基础上, 构造了 20 种谱分布, 包括单峰和 双峰, 这取决于不同的谱参数, 不同的粒子大小和不同的云的冰水含量( IWC). 其中, IWC 变化范围为 $3 \times 10^{-5} \sim 0.25 \mathrm{gm}^{-3}$.

对非球形冰晶粒子的形状考虑了两种: 六角柱状和六角盘状. 对于这两种形状冰晶尺度 分布的一个重要特征参量 $r_{e}$ (有效半径) 来说, 对应上述 IWC 的变化范围, $r_{e}$ 的相应变化范围 分别为 30 $325 \mu_{\mathrm{m}}$ (六角柱状冰晶) 和 $42 \sim 449 \mu_{\mathrm{m}}$ (六角盘状冰晶). 
同时考虑以下 4 个波长, 即 $1.38,3.979,6.5$ 和 $11.03 \mu_{\mathrm{m}}$, 其中, 3.979 和 $11.03 \mu_{\mathrm{m}}$ 为 目前卫星遥感常用的并分别位于 3.8 4 和 $8 \sim 12 \mu_{\mathrm{m}}$ 的大气红外窗区通道; 1.38 和 $6.5 \mu_{\mathrm{m}}$ 分别位于近红和红外水汽吸收带.

冰晶粒子的单次散射用改进的射线光学理论 ${ }^{[4,5]}$ (当 $L>45 \mu_{\mathrm{m}}$ ) 和 $\mathrm{M}$ ie 散射理论(当 $L$ $\left.\leqslant 45 \mu_{\mathrm{m}}\right)$ 计算出高斯节点的相函数元, 散射截面和衰减截面, 然后对尺度分布积分即得出卷 云体积散射系数 $\beta_{s}$. 体积衰减系数 $\beta_{e}$ 和相函数 $P_{11}(\theta)$. 由相函数的一阶矩得出不对称因子 $g, \beta_{s}$ 与 $\beta_{e}$ 之比是单次散射反照率 $\omega_{0}$. 利用 $\omega_{0}$ 和 $g$ 进而计算出 “相似性参数” ${ }^{[6]} S$. 卷云的 光学厚度 $\tau_{c}$ 则由体积衰减系数对卷云垂直方向积分得出. 卷云底高 $9 \mathrm{~km}$, 云厚 $2 \mathrm{~km}$.

假设云层为平面平行、水平均匀而垂直非均匀, 采用改进的两层冰云模式 ${ }^{[7]}$, 则冰晶粒 子的多次散射和辐射传输特性用累加-倍加法 ${ }^{[8]}$ 进行计算.

图 1 表明, 在所考察的波长中, 只有当 $\lambda=1.38 \mu_{\mathrm{m}}$ 时, 相似性参数随着冰晶粒子有效半 径 $r_{e}$ 增大而有规则地增大, 显示出 $S$ 对 $r_{e}$ 具有良好的依赖关系, 从而预示 $1.38 \mu_{\mathrm{m}}$ 波长可 用于探测卷云微物理学特性的潜在可能性. 应该指出, 上述两个大气红外窗区通道以及 6.3 $\mu_{m}$ 水汽吸收带不具备上述良好的特性.

光学厚度 $\tau_{c}$ 是决定卷云中辐射传输过程的一个基本参量, 不对称因子 $g$ 和单次散射反 照率 $\omega_{0}$ 有时也起着重要的作用. 而 $g$ 是相函数的一阶矩, 它跟其他散射参数一样, 不仅取决 于入射波波长，而且还依赖于冰晶粒子的形状及其尺度分布 ${ }^{[9]}$. 图 2 和图 3 进一步给出 1.38 $\mu_{\mathrm{m}}$ 波长卷云的多次散射特性, 即透过率(图 2) 和反射率(图 3) 随光学厚度 $\tau_{c}$ 的变化特征.

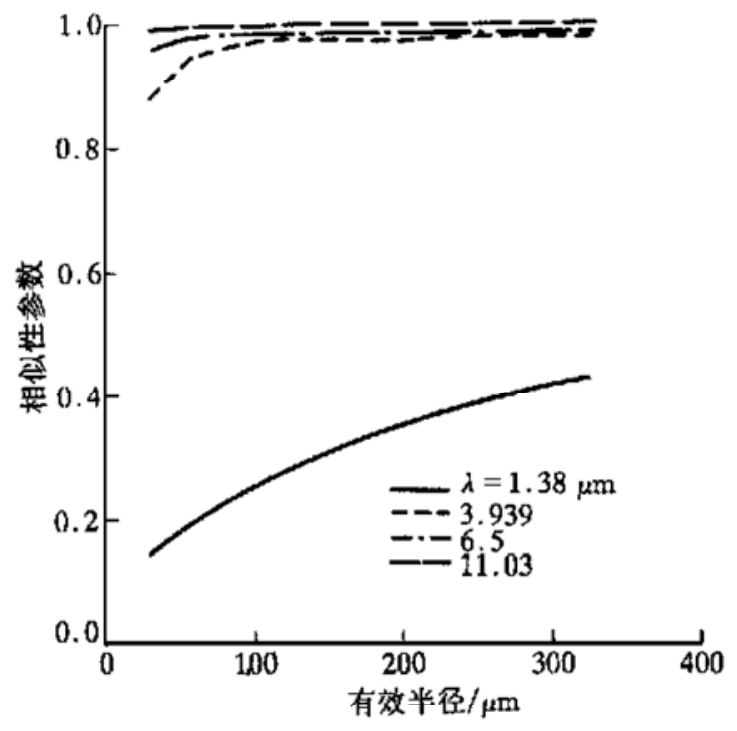

图 1 卷云在 $1.38 \mu_{\mathrm{m}}$ 和其他波长的相似性参数 与有效半径的关系

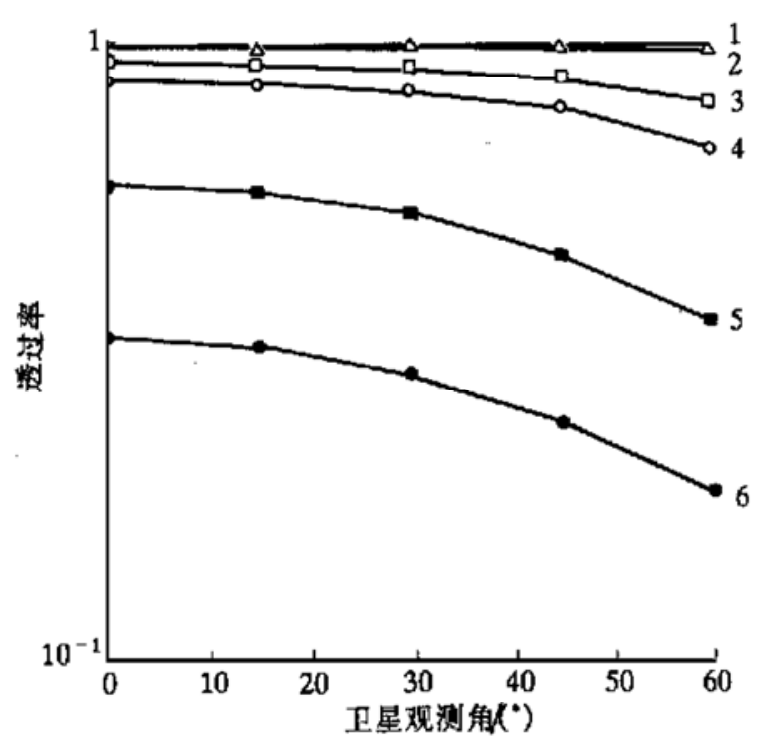

图 2 具有不同光学厚度的卷云透射率随卫 星观测角的变化 $\left(\lambda=1.38 \mu_{\mathrm{m}}\right)$

$1-\tau=0.55,2 \longrightarrow 0.10,3-0.88,4-1.63$,

$5-4.34,6-8.05$

由图 2 可见, 变化冰晶粒子的谱分布, 使 $\tau_{c}$ 由小变大; 随着 $\tau_{c}$ 的增大, 卷云中冰晶粒子 的透射率有规则的减小. 应该指出, 在所考察的波长中, $\lambda=1.38 \mu_{\mathrm{m}}$ 最透明, 即使 $\tau_{c}$ 增大到 
8.05 , 当卫星观测角 $\theta \leqslant 30^{\circ}$ 时，仍有 $T_{\mathrm{c}} \geqslant 0.3$. 而 $\lambda=11.03 \mu_{\mathrm{m}}$, 当 $\tau_{c}>8$ 时, $T_{\mathrm{c}}$ 只有 $10^{-3}$ 量 级(图省略). 这主要是因为冰在 $1.38 \mu_{\mathrm{m}}$ 的复 折射率的虚部很小, 卷云吸收很弱, 所以有更多 的透射分量, 而且, $g$ 与其他波长相比略小, 因 而又可有较多的反射分量.

图 3 表明, 对 $\lambda=1.38 \mu_{\mathrm{m}}$, 随着 $\tau_{c}$ 的增大, 反射率依次从 $10^{-3}$ 量级增大到 $10^{-1}$ 量级. 卷云 的 $R_{\mathrm{c}}$ 在所考察的其他波长上比 $1.38 \mu_{\mathrm{m}}$ 要小一 个数量级以上(图省略). 而且, 在所考察的波长 中, 只有在 $1.38 \mu_{\mathrm{m}}$ 波长上 $R_{\mathrm{c}}$ 随 $\tau_{c}$ 的变化才有 如图 3 所示那样的规律. 图 2 和图 3 显示了利用 $1.38 \mu_{\mathrm{m}}$ 水汽吸收带探测卷云光学厚度的潜在 应用前景. 应该指出, 由于大气中的水汽主要集 中在对流层下层, 地球表面对太阳短波辐射的反 射将被 $1.38 \mu_{\mathrm{m}}$ 吸收带所吸收, 因此, 空间遥感

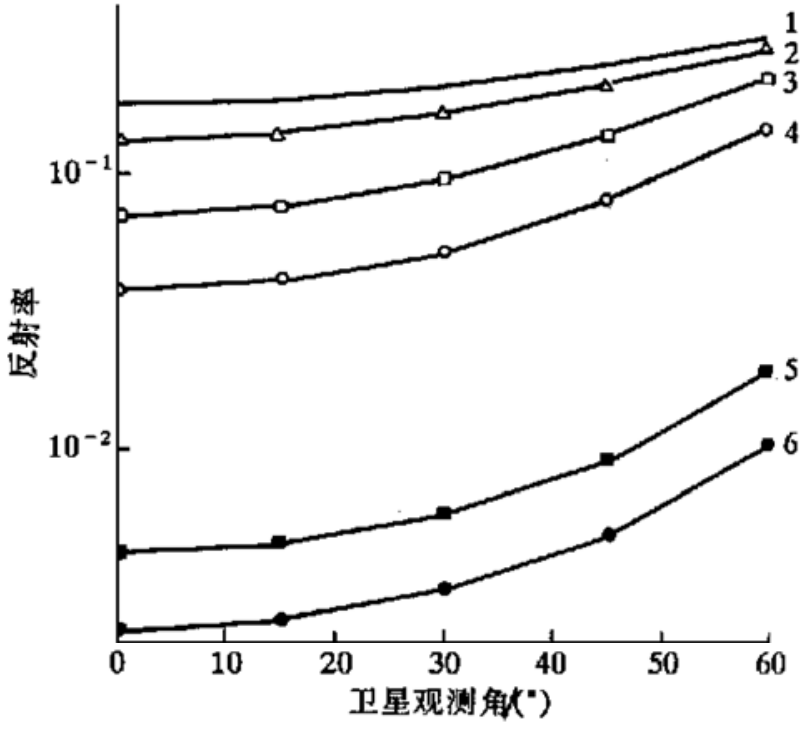

图 3 具有不同光学厚度的卷云反射率 随卫星观测角的变化 $\left(\lambda=1.38 \mu_{m}\right)$ 图中 1 6 注解同图 1 器( 辐射计) 探测到的能量主要来自于中层水云和高层冰云的散射, 故即使在大陆上空的薄卷 云, 利用 $1.38 \mu_{\mathrm{m}}$ 波长也容易被探测到. 本文初步揭示的 $1.38 \mu_{\mathrm{m}}$ 的良好特性, 显示了它作 为新的卫星遥感通道, 探测卷云微物理特性和光学特性的潜在可能性.

致谢 本工作为国家自然科学基金(批准号: 49675255)、北京大学暴雨监测与预测国家重点实验室(LSSR) 和 中国科学院大气物理研究所中层大气与全球环境探测开放实验室(LAGEO) 等课题资金的资助项目.

\section{参 考 文 献}

1 Gao B C, Kaufman Y J. Selection of the 1. $375 \mu_{\mathrm{m}}$ M ODIS channel for remote sensing of cirrus clouds and stratospheric aerosols from space. J Atmos Sci, 1995, 52: 4 231 4237

2 Hutchison K D, Choe N. A quantitative assessment on the value of 1.38 micron imagery for the automat ed analysis of optical ly_thin cirrus in daytime imagery. In Proc Europto Series, 1995, SPIE 2578: 53 60

3 NASA. 1995 MTPE/EOS Reference Handbook, NASA_GSFC, 1995

4 Zhang J, Xu L. Light scattering by absorbing hexagonal ice crystals in cirrus clouds. Appl Opt, 1995, 34: 5 867 5 874

5 Xu L, Zhang J. Light scattering by randomly oriented hexagonal ice crystals: improved diffraction computations and diffraction properties of ice crystals. Optik, 1996, 101: 161 165

6 Van de Hulst H C Multiple Light Scattering, Tables, Formulas, and Applications. Vol 2. New York: Academic Press, 1980

7 Xu L, Zhang J. Simulation of retrieval of the base height and thickness of cirrus clouds from satellite data. J Opt Soc Am A, 1992, 9: $1536 \sim 1546$

8 Lenoble J. Radiative Transfer in Scattering and Absorption Atmospheres: Standard Computational Procedures. Hampton: A Deepak, 1985

9 Xu L. Zhang G, Ding J, et al. Light scattering by polydispersions of randomly oriented hexagonal ice crystals: phase function analyses. Optik, 1997, 106: 103 114 